Annuaire suisse de politique de développement

3 | 1983

Annuaire Suisse - Tiers Monde 1983

\title{
Les finances fédérales et l'aide publique au développement de la suisse
}

Jacques Martin

\section{(2) OpenEdition}

Édition électronique

URL : http://journals.openedition.org/aspd/1021

DOI : 10.4000/aspd.1021

ISSN : 1663-9669

Éditeur

Institut de hautes études internationales et du développement

\section{Édition imprimée}

Date de publication : 1 janvier 1983

Pagination : 215-226

ISSN : 1660-5934

\section{Référence électronique}

Jacques Martin, «Les finances fédérales et l'aide publique au développement de la suisse », Annuaire suisse de politique de développement [En ligne], 3 | 1983, mis en ligne le 29 novembre 2012, consulté le 08 septembre 2020. URL : http://journals.openedition.org/aspd/1021 ; DOI : https://doi.org/10.4000/ aspd. 1021 


\title{
LES FINANCES FEDERALES ET L'AIDE PUBLIQUE AU DEVELOPPEMENT DE LA SUISSE
}

\author{
Jacques Martin
}

\begin{abstract}
Zusammenfassung: Die Bundesfinanzen und die öffentliche Entwicklungshilfe der Schweiz
\end{abstract}

Mit 75 Franken pro Jahr und pro Einwohner ist die Schweizer EntwickLungshilfe im Verhältnis zu der der anderen OECD-Länder auch 1982 eine der niedrigsten. In Anbetracht der umfassenden Ausgabenkürzungen im Budget und in der Finanzplanung des Bundes bleibt kaum zu erwarten, dass sich diese Situation bald ändern wird. Der vom Bund oft zur Schau gestellte politische Wille, die öffentliche Entwicklungshilfe den Durchschnittswerten der OECD anzugleichen, wird durch die Umstände widerrufen.

Auch wenn die aktuelle Wirtschaftskrise dafür nicht die Hauptverantwortung trägt, so schafft sie doch ein Klima in der Öffentlichkeit und in der Regierung, welches kaum dazu beiträgt, nach Lösungen zu suchen, die den Bedürfnissen der Entwicklungsländer Rechnung tragen. Darüberhinaus läuft die Schweizer öffentliche Entwicklungshilfe, die vor allem den Ärmsten zukommt, Gefahr, einen ihrer Vorzüge zu verlieren, nämlich nicht an die Lieferung von Schweizer Gütern gebunden zu sein.

Der vorliegende Artikel befasst sich mit zwei Fragen: Wird die öffentliche Entwicklungshilfe, wie vom Bundesrat während der letzten Jahre mehrmals erklärt, weiterhin eine vorrangige Stellung einnehmen? Ist est möglich, sie unter den vorherrschenden Bedingungen ohne zusätzliche Steuereinnahmen zu erhöhen?

\section{INTRODUCTION}

L'opinion prévaut, à juste titre, que la révision vers le bas des objectifs d'aide publique au développement (APD), que s'était fixés le Conseil fédéral pour la première moitié environ des années 80 , est une conséquence directe de la dégradation de la situation des finances fédérales. II serait faux, par contre, de chercher à voir dans la seule crise économique actuelle et ses problèmes connexes les raisons ayant conduit à 
cette situation: la genèse du déséquilibre budgétaire fédéral est en partie antérieure au début des années 70 .

Toutefois, le débat politique et public (au Parlement, dans la presse, dans l'opinion) sur les remèdes à apporter à cette situation a largement été influencé, depuis 1980-1981, par le climat économique dégradé (légèrement, pour ce qui est de la Suisse) ainsi que par la philosophie défendue actuellement avec une nouvelle vigueur par certains milieux tenants du "moins d'Etat». Ce climat et l'expression de ces opinions $\mathrm{n}^{\prime}$ ont pas été sans influencer le Parlement et l'Exécutif fédéral; ils mettent en outre bien en évidence les limites de la volonté politique réelle de consacrer des moyens accrus à I'APD ou, pour le moins, la fragilité de cette volonté.

Tout se passe donc comme si la Suisse n'était prête à s'accorder les moyens de la politique de solidarité et d'ouverture qu'elle proclame, que dans la mesure où les sacrifices que cela exige par ailleurs ne sont pas ressentis comme tels! Dans ces conditions, l'accroissement de I'aide publique au développement est-il encore une priorité ?

Dans ce papier, nous chercherons à voir pourquoi et par qui les crédits d'APD suisse, dont le Gouvernement lui-même prévoyait une augmentation substantielle il y a encore deux ou trois ans, se sont vus amputés, pour les années 1981 à 1987, de quelques bonnes centaines de millions de francs par rapport aux diverses prévisions. L'effort envisagé pour que la Suisse atteigne enfin, vers la moitié de la décennie, la moyenne des prestations d'APD des pays de I'OCDE $(0,35 \%$ du PNB) $n$ 'est finalement pas fourni. II est donc à craindre que notre pays reste ainsi jusque vers la fin de la décennie à la traîne des pays développés. Au moment où paraît cet article, toutefois, un léger déblocage de la situation a eu lieu et la menace d'un gel de l'APD à 0,26\% du PNB pour les années 1984 à 1986 a peut-être été écartée. Par rapport aux plans financiers antérieurs pour ces années, les allocations budgétaires envisagées ont néanmoins été assez sensiblement réduites. Les pays en développement, notamment les plus pauvres dont les besoins vont croissant et vers lesquels une bonne partie de I'APD suisse se dirige, risquent ainsi de devoir se contenter de programmes quasi-stagnants en valeur réelle pour plusieurs années encore.

\section{MECANISMES DES FINANCES FEDERALES}

II ne semble pas inutile de rappeler quelle est la procédure suivie pour la mise à disposition par la Confédération des crédits d'APD. Ces mécanismes sont brièvement décrits ci-dessous dans le but de camper le décor et d'approcher la réalité juridique et technique des mesures prises, ces dernières années, en vue de limiter l'augmentation des allocations budgétaires pour I'APD. 
A l'occasion du début de chaque nouvelle législature (période de 4 ans), l'Exécutif fédéral présente au Parlement un document intitulé «Rapport sur les grandes lignes de la politique gouvernementale» dans lequel, dans tous les domaines de sa compétence, le Conseil fédéral fixe ses intentions. II s'agit surtout d'objectifs généraux, parfois chiffrés (au niveau des ordres de grandeur). Ces grandes lignes, préparées par l'Administration, sont présentées au Parlement qui en prend connaissance, y consacre un débat mais n'a pas à les approuver. La discussion aux Chambres peut, bien entendu, influencer les orientations indiquées.

En se basant sur la loi fédérale du 19 mars 1976 sur la coopération au développement et l'aide humanitaire internationales, le Conseil fédéral présente à ce même Parlement des demandes de crédits de programme, pluriannuels dans la règle (appelés parfois crédits-cadres), dont I'approbation revient à autoriser l'engagement (1) des moyens financiers nécessaires à la mise en œuvre de mesures d'APD. Ces crédits-cadres font l'objet $d^{\prime} u n$ message aux Chambres dans lequel les intentions générales sont explicitées et des objectifs opérationnels plus ou moins précis indiqués; les sommes nécessaires aux engagements proposés sont indiquées également (montants globaux). Cette procédure donne l'occasion au Parlement d'aborder, de manière thématique, une discussion de fond sur I'APD. L'approbation du Parlement ne constitue toutefois pas un aval budgétaire.

En effet, les dépenses annuelles afférant aux programmes d'APD doivent encore être discutées et approuvées à l'instar de toute autre dépense publique. L'Exécutif s'est doté d'un outil de planification pluriannuelle: le plan financier, qui indique les intentions de dépenses pour les trois années suivantes. Ce plan est présenté au Parlement, mais n'est pas soumis à son approbation formelle; il n'indique d'ailleurs que les tendances. C'est le budget annuel (pour l'année suivante) qui est soumis aux Chambres pour approbation lors de chaque session d'hiver.

En conclusion: c'est le budget annuel qui représente l'instrument légal d'octroi, à l'Exécutif, des fonds nécessaires aux tâches envisagées et le seul qui lui confère l'autorisation de les dépenser. Les étapes précédentes sont les instruments de la politique ou de la planification financière.

\section{HISTORIQUE DE LA DETERIORATION DES FINANCES FEDERALES}

La genèse des difficultés que connaissent les finances fédérales se situe au début des années 70; c'est à compter de 1975, toutefois, que I'on observe un déséquilibre croissant entre les recettes et les dépen- 
ses. Jusqu'alors, au cours de son histoire, l'Etat fédéral moderne avait toujours veillé à limiter aux situations extraordinaires ses entorses aux principes de l'orthodoxie budgétaire (par exemple au cours des guerres mondiales), car le sentiment prévaut en Suisse que l'Etat doit gérer son budget "comme une bonne ménagère", c'est-à-dire ne pas dépenser plus que ce dont il dispose. Pour mémoire, il faut rappeler que la structure fédéraliste de la Suisse veut que le budget fédéral (c'est-à-dire de l'Etat central) ne représente qu'une partie seulement, relativement restreinte (environ 35 à $40 \%$ ), de l'ensemble des budgets publics suisses: les cantons et les communes lèvent également l'impôt et assument une bonne part des dépenses publiques.

Les causes du déficit fédéral tiennent en fait simplement à une explosion des dépenses sans qu'il y ait eu, du côté des recettes, d'accroissement parallèle. Même si la situation de la Suisse se compare positivement à celle d'autres pays développés, voyons de plus près les causes de ce déficit chronique. Accompagnant le développement socioéconomique, les activités de l'Etat moderne s'amplifient et les tâches qui lui sont confiées s'étendent, se diversifient et se compliquent. La Suisse, malgré sa structure fédéraliste, n'a pas échappé à ce processus. Le développement des tâches de l'Etat social (sécurité sociale, assurance-maladie, etc.), aussi souhaitable qu'ait pu être cette évolution, ainsi que l'adjonction de nouvelles tâches et l'expansion de tâches existantes provoquèrent des charges de plus en plus lourdes alors que, parallèlement, I'une des sources de recettes traditionnelles de la Confédération, les droits de douanes, tendait, notamment en raison des accords avec l'AELE puis la CEE, à se tarir; les recettes douanières qui représentaient encore $32 \%$ des recettes totales de la Confédération en 1960 n'en représentaient plus que $11,2 \%$ en 1981 (2). Cette baisse des recettes est due aussi au fait que les droits sont perçus, en Suisse, de manière spécifique et non ad valorem, ce qui empêche une indexation automatique compensant I'inflation. Au cours de toutes ces années, toutefois, augmentait le rendement de l'impôt fédéral direct (ou impôt fédéral pour la défense nationale $=$ IDN), en raison de la progression à froid; il assure désormais près de $20 \%$ des recettes totales (1960: 13,5\%).

Parmi les tâches nouvelles figure justement l'aide publique au développement qui commença à prendre une certaine importance en Suisse il y a vingt ans seulement, Certes, au cours de la période de haute conjoncture durant laquelle nombreux étaient ceux qui vaquaient aux affaires à un rythme effréné, voire malsain (ne parlait-on pas en Suisse de "surchauffe"?), le projecteur de l'actualité politique n'était guère braqué sur les finances fédérales, dont la santé ne fournissait pas de raison d'inquiétude majeure. D'ailleurs, notamment chez les fédéralistes, les détracteurs de l'augmentation des dépenses publiques fédérales veillaient au grain, même s'ils ne purent que freiner modestement le développement des activités de l'Etat, ergo les dépenses. 
Les premières difficultés sérieuses coïncident plus ou moins avec la fin de cette période euphorique de croissance sans limites et avec le premier réajustement des prix par les producteurs de pétrole.

La dette de la Confédération augmentait soudain à un rythme soutenu (elle avoisine actuellement $\mathbf{2 0}$ milliards de francs et son service coûte plus d'un milliard par an) et les budgets présentés devenaient systématiquement déficitaires (ordre de grandeur: au-delà du milliard de francs d'excédent de dépenses par rapport aux recettes). Ce déséquilibre budgétaire étant très mal considéré, surtout par la droite (la gauche, quant à elle, étant encline à préférer cette situation à un démantèlement des acquis sociaux), ceux qui souhaitaient voir limitée ce qu'ils considèrent comme une emprise grandissante de l'Etat dans les affaires du pays furent trop contents de pouvoir exiger un retour dans un délai raisonnable au sacro-saint équilibre . (Deux rappels, à ce stade: d'une part, la Constitution fédérale prévoit, à son article 42 bis, introduit en 1958, que le Conseil fédéral veille à amortir le découvert de son bilan, c'est-à-dire non seulement à éviter un déficit budgétaire, mais encore à diminuer sa dette. D'autre part, il semble intéressant de relever que la dette publique de la Confédération, exprime en pourcentage du PNB, tend à diminuer; ainsi ce rapport qui était de 20,2\% en 1960 n'est plus que de $13,8 \%$ en 1980 et de $12,1 \%$ en 1982 ; de même, si l'on compare entre eux les intérêts passifs payés pour cette dette et les rentrées fiscales de la Confédération, l'on s'aperçoit que le rapport tend actuellement à diminuer: de $7,8 \%$ en 1960 à $6 \%$ en 1980 et $5,9 \%$ en 1982 (3).)

Le peuple suisse, consulté, ayant refusé à plusieurs reprises des impôts nouveaux (les cas les plus notables étant les deux refus successifs de l'introduction de la TVA, à la fin des années 70), la seule issue, sous la pression de plus en plus forte d'une majorité des parlementaires, consista à freiner l'augmentation du volume des dépenses.

Dans le chapitre suivant, nous allons voir comment les mesures proposées ont affecté les crédits d'aide publique au développement (APD) que la Suisse envisageait de consacrer à cette tâche importante.

\section{LE FREIN A L'ACCROISSEMENT DE L'APD OU LES ENGAGEMENTS RENIÉS}

"A diverses reprises dans le passé [...] le Conseil fédéral a estimé que I'aide suisse au développement était insuffisante et qu'il entendait s'efforcer de la porter [...] à $0,35 \%$ du produit national brut suisse. Lors de l'examen de la motion parlementaire de 1981 sur le plan financier (4), le Conseil fédéral est arrivé à la conclusion que les objectifs voulus par la motion n'étaient pas compatibles avec la volonté d'accroître I'aide publique au développement [...]. Le Conseil fédéral 
se voit dès lors contraint de renoncer à poursuivre son effort en la matière (5). " (Réponse du Conseil fédéral du 28 janvier 1983 à l'interpellation Jean Ziegler (PS/GE) du 14 décembre 1982.)

Ainsi le Parlement et l'Exécutif, soucieux de remettre de l'ordre dans la maison fédérale, s'en sont-ils pris aux dépenses afin d'arriver à équilibrer le budget de la Confédération (ou pour le moins réduire drastiquement son déficit) avant même la fin de la décennie. Tout un train de mesures a été imaginé à cet effet.

La question, dès lors, se posait pour l'Administration du choix d'un ordre de priorité au sein des différentes dépenses: auxquelles s'attaquer et de combien les diminuer? En d'autres termes, quelles seront les cibles? La chose est d'autant moins aisée que, dans un Etat moderne, la croissance de nombreuses dépenses revêt un caractère automatique (par exemple: I'indexation des salaires ou bien la sécurité sociale) et qu'il est difficile d'y toucher sans modifier leur(s) base(s) légale(s), parce que cela prend du temps et n'est pas toujours politiquement souhaitable.

Le Conseil fédéral a donc proposé deux types de mesures: certaines faisant appel à la "symétrie des sacrifices" (en l'occurrence: coupures de $10 \%$ dans tous les groupes de tâches); d'autres, orientées vers des cibles précises, dans le cadre de mesures d'assainissement affectant les plans financiers établis pour plusieurs années.

L'APD fut une victime facile de cette deuxième série de coupures, malgré les louables efforts de certains parlementairs de gauche et du centre ainsi que de quelques députés isolés de la droite libérale. La discussion de l'été 1983 a montré qu'une partie du Parlement réalisait la gravité des coups de frein donnés (et qu'il était envisagé de donner encore), à l'accroissement nécessaire et déjà proclamé des dépenses d'APD. Cette prise de conscience lappuyée par une opinion publique qui n'a pas hésité à adresser une pétition au Conseil fédéral munie de plus de 200.000 signatures) aura porté quelques fruits, l'Exécutif fédéral s'étant montré prêt à revenir (certes prudemment) sur sa décision de gel de I'APD.

La suppression ou la diminution d'un certain nombre de subventions représentent en fait un transfert de charges aux cantons, voire aux collectivités locales. L'on comprend qu'il ne peut en aller ainsi de I'APD qui fait partie des tâches propres de la Confédération, dans le cadre de ses relations avec l'étranger, même si, occasionnellement, certains contons et communes (et c'est heureux!) consentent aussi à un effort en faveur des pays en développement.

Malgré les "Grandes lignes de la politique» et les objectifs qui y sont définis, malgré les crédits de programme dûment approuvés par les deux Chambres, malgré les plans financiers (de 1979, de 1980 et même du 5 octobre 1981 (6)), I'APD restait une cible toute désignée: 
en effet, aucune obligation contractuelle n'existe, pendant les mois, voire les années, que durent la préparation et la négociation d'un projet de coopération technique ou $\mathrm{d}^{\prime}$ aide financière. Juridiquement, donc, les coupures ne soulèvent normalement pas de problèmes pour ce qui est des projets dans le pipe-line! Et puis, les principaux intéressés sont bien lointains et ne peuvent guère faire valoir leurs vues et défendre leurs projets; et ce ne sont pas des électeurs!

En autorisant un certain espoir à différents stades du processus de planification et de la discussion politique intérieure ainsi que dans le dialogue international, puis en limitant les moyens financiers nécessaires, le gouvernement du pays pratiquement le plus riche du monde s'est contredit et son crédit moral ne devrait pas en sortir indemne. Que le Parlement et le peuple portent leur part de responsabilté n'y change rien: les ordres de priorité que l'on sait ont été établis par le Conseil fédéral. Ce dernier se trouve bien sûr confronté à un dilemme, les charges augmentant et les moyens ne lui étant pas accordés pour y faire face. II est fort probable que parmi les conseillers fédéraux les opinions divergent quant aux remèdes à apporter à cette situation; mais le Conseil fédéral, en la matière, a choisi de donner suite aux injonctions du Parlement.

\section{LES CONSEQUENCES POUR LA COOPERATION SUISSE AU DEVELOPPEMENT DU RALENTISSEMENT DE LA CROISSANCE DE L'APD}

Nous l'avons montré, la situation décrite n'est pas seulement le résultat de coupures budgétaires à caractère technique, mais bien plus celui d'un certain climat politique qui a permis qu'elles se fassent.

Pour ce qui est de l'impact des mesures prises sur le volume de I'APD suisse pour les années 1981-1987, nous renvoyons le lecteur aux chiffres figurant au tableau de l'Annexe 1. L'Annexe 2 offre un survol des apports d'APD de la Suisse depuis 1961 et montre la progression très lente de cette aide publique au cours de ces vingt et quelques années, particulièrement en termes réels.

Le tableau de l'Annexe 1 montre également l'ampleur de la série de mesures prises ou qu'il est envisagé de prendre à propos de l'APD: si le gel est évité de justesse, la progression reste bien en deçà des prévisions (et des engagements) antérieurs.

Conséquence de ces réductions budgétaires: la qualité de la coopération est susceptible d'être affectée également. Par exemple, le dialogue avec le partenaire est rendu difficile et beaucoup d'énergie est absorbée par les services concernés pour de délicats exercices de programmation et de calibrage des moyens au plus juste, au risque de priver les projets d'une certaine flexibilité. Depuis de nombreuses années 
enfin, le blocage du personnel fédéral (autre conséquence de la situation des finances fédérales) fait problème, en particulier pour la DDA qui, bon an mal an, doit faire face à des tâches croissantes.

Quant à savoir si, selon la question Blunschy (PDC/SZ) au Conseil national), "les mesures d'économies prises [...] ont entraîné une réduction affectant des projets en cours $[\ldots]$ ou si elles ont forcé à différer, voire refuser, l'exécution de tâches nouvelles", le Conseil fédéral a répondu le 28 janvier 1983:

- qu'il ne se produisait guère de cas où une action en cours a dû être interrompue ou un projet prêt à être mis en œuvre refusé, mais que, par contre:

- on avait dû renoncer à de nombreux projets et opposer des refus à de nombreuses demandes, alors même qu'ils correspondaient à des besoins urgents des populations les plus pauvres.

A ce jour, la DDA sait, par exemple, qu'elle ne peut consacrer qu'environ $10 \%$ seulement de ses moyens à commencer de nouvelles actions, $90 \%$ devant être affectés à la poursuite de projets en cours (y compris de nouvelles phases).

Autre résultat dû cette fois, en partie du moins, à la crise économique : nombre d'industriels et de bureaux privés souhaitent désormais pouvoir participer de manière plus active que par le passé aux activités de coopération au développement. Cela tient probablement aussi à l'importance croissante, au fil des années, des moyens financiers à la disposition de la Confédération pour la coopération au développement. Certes un recours accru au secteur privé peut être souhaitable, voire représenter, dans certains cas, la meilleure solution à un problème opérationnel; mais l'intérêt de ces milieux (activement soutenus par leurs lobbies) pourrait bien avoir des conséquences sur l'actuel taux de déliement de I'APD suisse, par exemple en provoquant une tendance à l'augmentation de la part des crédits mixtes dans le total de I'APD. Rappelons que dans le cadre des mesures de relance de l'économie suisse, de mars 1983, une augmentation de 350 millions de francs pour les mesures économiques et commerciales au titre de la coopération internationale au développement a été accordée; cette aide est par définition liée à la fourniture de biens et de services suisses.

$D^{\prime}$ autres risques découlant de la situation économique internationale pourraient se présenter encore: ainsi l'octroi d'aides "préventives" (crédits mixtes, aide à la balance des paiements) à des pays dont l'économie chancelante et la situation politique intérieure dégradée font courir le risque de les voir "basculer" politiquement dans une direction opposée aux intérêts des pays occidentaux (exemple récent de la Turquie); ou encore un retour systématique à l'aide liée même pour les crédits $d$ 'aide financière bilatérale. 
Conséquences psychologiques et culturelles enfin, au niveau de l'opinion publique. II ne fait guère de doute que, en présence du chômage et d'une certaine désindustrialisation de diverses régions, les personnes touchées, de près ou dans leur entourage, tendent à voir dans l'aide au développerment un luxe de peu de priorité face à la relance économique intérieure. Le concept de solidarité internationale est plus difficile à faire passer, même - et surtout - chez les travailleurs touchés par la crise.

\section{CONCLUSIONS}

Cette étude des décisions prises en matière d'APD vise à démontrer que si la crise économique actuelle $n^{\prime}$ est pour rien dans la détérioration des finances fédérales, elle n'en affecte pas moins la recherche de solutions: celles-ci ont finalement pris la forme d'une révision des ordres de priorités:

I'aide au développement, pour le Conseil fédéral, ne revêt en fait pas une priorité qui soit véritablement de premier plan!

Dès lors, que penser du discours de notre Exécutif dans les enceintes internationales? Que penser de la prise de conscience, officiellement déclarée, des réalités de l'interdépendance dont l'importance a été maintes fois répétée et l'a été encore à la CNUCED VI?

Certes, le Conseil fédéral n'est pas seul en cause; il y a le Parlement et le peuple et ce devrait être à ces derniers de reconnaître une fois pour toutes la nécessité de donner au Gouvernement les moyens de sa politique: comme une révision des priorités au sein des tâches fédérales semble difficile, il n'y aura de véritable augmentation de l'aide publique au développement qu'en relation avec une augmentation des recettes fiscales de la Confédération.

Des limites sont en effet atteintes en matière d'économies; quant à l'exercice d'assainissement en cours (sur le succès duquel des hommes politiques ont émis des doutes), pour méritoire qu'il soit, il ne témoigne d'aucune vue d'avenir sur l'importance d'une augmentation substantielle de I'APD. Que se passera-t-il après 1986: continuera-ton à mesurer chichement I'APD de la Suisse aux pays les plus pauvres, en la maintenant à moins de Fr. 100. - par an et par habitant ? Que fera la Suisse dans les années 90 ? Quelles sont les perspectives?

Soyons nets: si de nouvelles recettes représentent le seul moyen réaliste d'augmenter l'APD, il importe que tout pétitionnaire, motionnaire ou interpellateur, tout groupement "tiers-mondiste», tout citoyen en soit conscient. En effet, de nouveaux impôts seront probablement nécessaires et, le cas échéant, il conviendra de ne pas s'y opposer. 


\section{Notes}

1. Engagement: une décision de consacrer, pour un objectif et avec des moyens bien précis, une somme déterminée en vue de la réalisation d'un projet ou du versement d'une contribution; l'engagement est suivi de versement(s) - paiement(s) - éventuellement échelonnés sur plusieurs années.

2. Sans les droits supplémentaires sur les carburants.

3. Il est vrai que vers la fin de la période de haute conjoncture ces rapports étaient encore plus faibles ( $8 \%$ et 3,6\% respectivement en 1970 ).

4. II s'agit d'une motion parlementaire (M 80.052) exigeant des mesures sérieuses d'assainissement des finances fédérales.

5. C'est nous qui soulignons.

6. Ce dernier cas pour les années 1984 à 1986 .

\section{Sources}

Comptes d'Etat de la Confédération (diverses années).

Annuaire statistique de la Suisse 1982.

Plans financiers 1984-1986.

Bulletins officiels de l'Assemblée fédérale.

Divers documents et rapports de la DDA.

L'auteur tient à remercier ici Mme Catherine Graf du Service de statistique de la DDA de son appui dans la collecte et l'interprétation des informations chiffrées sur les coupures opérées au sein des finances fédérales. 


\section{COUPURES ET MESURES D'ASSAINISSEMENT AFFECTANT L' A.P.D. DE LA SUISSE}

DECISION

Type de

Année

affectée

Type de
décision

(base légale)

BUT ET AMPLEUR

Technique de réduction

A.F. 20.6 .80

Réduction linéaire de $10 \%$

Ajustement (y-c. aide

1981

A.F. 15.6 .81

économique à la Turquie)

1982

A.F. 20.6 .80

Réduction linéaire de $10 \%$

Réduction suppl. s/budg. 82

Réduction suppl. s/budg. 82

Réduction linéaire de $10 \%$

Prorogation réduct.linéaire

(modification A.F. 20.6.80)

Propositions d'assainissement

(rép. à motion parlementaire)

$$
\begin{aligned}
& \text { Décision du } \\
& \text { C.F. } 04.10 .82
\end{aligned}
$$

1985

1986

Diverses mesures d'assainissement déjà prises et/ou actuellement en discussion

1987

Notes: * Chiffres estimatifs

* * N'a pas fait l'objet d'une décision dans le cadre du plan financier
CONSEQUENCES PAR RAPPORT AUX

PLANS FINANCIERS (P.F.)

P.F. original Réduction Budget

(date) ajustement P.F.final

(mio Fr) (mio Fr) (mio Fr)

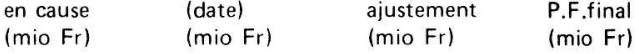

487

$-49$

438

$(27.12 .79)$

$+20$

- 56

591

$-56$

535

(08.09.80)

$-10$

$-40$

- 58

609

$-50$

08.09 .80

- 58

485

710

$-71$

639

(05.10.81)

.50

800

$+150^{*}$

589

(27.12.79)

$\pm 900^{* *} \quad \pm 250^{*}$

**

- *

$\pm 650^{*}$

$\pm 750^{*}$

Sources: DDA et DFF 
ANNEXE 2

EVOLUTION DE L'AIDE PUBLIQUE AU DEVELOPPEMENT DE LA SUISSE DE 1961 A 1982

\begin{tabular}{|c|c|c|c|c|}
\hline ANNEE & $\begin{array}{l}\text { MONTANT * } \\
\text { (mio Fr) }\end{array}$ & $\%$ DU PNB & $\begin{array}{l}\text { AUGMENTATION } \\
\text { nominale }(\%)\end{array}$ & $\begin{array}{l}\text { ANNUELLE } \\
\text { réelle }(\%) i\end{array}$ \\
\hline 1961 & 37,6 & 0,08 & - & - \\
\hline 1965 & 47,5 & 0,08 & $6,02^{* *}$ & $1,1^{* *}$ \\
\hline 1970 & 130,5 & 0,14 & $22,4^{* * *}$ & $17,8^{* * *}$ \\
\hline 1971 & 116,9 & 0,11 & $-10,4$ & $-17,9$ \\
\hline 1972 & 248,1 & 0,21 & 112,2 & 93,6 \\
\hline 1973 & 206,6 & 0,15 & $-16,7$ & $-23,0$ \\
\hline 1974 & 202,3 & 0,14 & $-2,1$ & $-8,6$ \\
\hline 1975 & 267,4 & 0,18 & 32,2 & 23,8 \\
\hline 1976 & 281,4 & 0,19 & 5,2 & 2,8 \\
\hline 1977 & 286,2 & 0,19 & 1,7 & 1,4 \\
\hline 1978 & 310,0 & 0,20 & 8,3 & 4,9 \\
\hline 1979 & 343,7 & 0,21 & 10,9 & 8,6 \\
\hline 1980 & 412,5 & 0,23 & 20,0 & 16,5 \\
\hline 1981 & 452,6 & 0,24 & 9,7 & 2,8 \\
\hline 1982 & $489,6^{* * * *}$ & 0,24 & 8,2 & 0,6 \\
\hline
\end{tabular}

Notes: $\quad *$ $\quad \mathrm{Y}$ compris l'aide publique des cantons et communes.

** Moyenne sur 4 ans (base 1961)

* Moyenne sur 5 ans (base 1965)

**** Dès 1982, y compris les frais administratifs de I'aide.

i Utilisation de l'indice implicite des prix du PNB (1970 égal à 100) 